\title{
¿Feminismo(s) en transición? Intereses de las mujeres, estado y política pública en la Venezuela bolivariana
}

\author{
Anais D. López C. \\ Centro de Estudios Latinoamericanos Rómulo Gallegos, Venezuela \\ anais.lopez22@gmail.com
}

\section{Resumen}

En los últimos 15 años Venezuela ha experimentado un proceso de transformaciones sociales y políticas, que de forma evidente ha impactado al Estado y la institucionalidad que este ordena. El proceso constituyente convocado en 1.999 por el recién electo presidente, Hugo Chávez, hizo posible que además de la consulta y la posibilidad de aportar con propuestas concretas a la redacción del nuevo texto constitucional, las personas, colectivos y movimientos se involucraran de forma directa en la construcción de una nueva institucionalidad y por ende en el diseño y ejecución de las políticas públicas a través de las cuales se daría vida a lo refrendado en la nueva constitución. Así, las organizaciones feministas y los movimientos de mujeres, experimentaron por primera vez en mucho tiempo la posibilidad de ser sujetos visibles no solo desde el reconocimiento de derechos hasta entonces postergados, sino también desde la participación activa y directa en la transformación social que ocurría en el país.

Ahora bien, la dinámica de esta participación ha sido un proceso lleno de tensiones y rupturas al interior de las organizaciones feministas y de mujeres, que en medio de debates feministas aún no resueltos sobre el orden, las formas y la priorización de ciertas reivindicaciones, plantea hoy la necesidad de realizar un balance sobre lo que ha sido realmente conquistado la última década, en el marco del proyecto político bolivariano de transición al socialismo.

Palabras clave: feminismo, mujeres al poder, visibilidad, reconocimiento, democracia femenina

\section{Feminism(s) in transition? Women's interests, state and public policy in Bolivarian Venezuela}

\begin{abstract}
In the last 15 years, Venezuela has undergone a process of social and political transformation, which has an evident impact on the State and its institutions. The constitutional process convened in 1999 by the newly elected President Hugo Chávez made possible that, in addition to consultation and the possibility of bringing concrete proposals to the drafting of the new constitutional text, people, groups and movements were directly involved in the construction of a new institutionality and therefore in the designing and execution of the public policies through which would be given life to the endorsed in the new constitution. Thus, feminist organizations and women's movements experienced for the first time in a long time the possibility of being visible subjects not only from the recognition of rights hitherto deferred, but also from active and direct participation in the social transformation that occurred in the country.

However, the dynamics of this participation has been a process full of tensions and ruptures within feminist and women's organizations, which, in the midst of unresolved feminist debates about the order, forms and prioritization of certain claims, raises today the need to take stock of what has been really achieved in the last decade, within the framework of the Bolivarian political project of transition to socialism.
\end{abstract}

Keywords: feminism, women in power, visibility, recognition, feminine democracy 
Losúltimos15años, Venezuelahaexperimentado un proceso de transformaciones sociales y políticas, que de forma evidente ha impactado al Estado y la institucionalidad que este ordena. El proceso constituyente convocado en 1.999 por el recién electo presidente, Hugo Chávez, hizo posible que además de la consulta y la posibilidad de aportar con propuestas concretas a la redacción del nuevo texto constitucional, las personas, colectivos y movimientos se involucraran de forma directa en la construcción de una nueva institucionalidad y por ende en el diseño y ejecución de las políticas públicas a través de las cuales se daría vida a lo refrendado en la nueva constitución. Así, las organizaciones feministas y los movimientos de mujeres, experimentaron por primera vez en mucho tiempo la posibilidad de ser sujetos visibles no solo desde el reconocimiento de derechos hasta entonces postergados, sino también desde la participación activa y directa en la transformación social que ocurría en el país.

Ahora bien, la dinámica de esta participación ha sido un proceso lleno de tensiones y rupturas al interior de las organizaciones feministas y de mujeres, que en medio de debates feministas aún no resueltos sobre el orden, las formas y la priorización de ciertas reivindicaciones, plantea hoy la necesidad de realizar un balance sobre lo que ha sido realmente conquistado la última década, en el marco del proyecto político bolivariano de transición al socialismo.

La relación entre los movimientos feministas, de mujeres y el Estado ha sido desde sus inicios una relación compleja, tensa; por un lado el Estado encarna el lugar de lo público, y el feminismo reclama desde sus orígenes que se reconozca la condición de ciudadanía a las mujeres, así como la aceptación del espacio privado como ámbito de acción política y de política pública, por lo que, de ninguna forma es correcto hacer una analogía entre el feminismo y lo privado, en la medida en que los problemas que este plantea sobre la posición de las mujeres en la sociedad no afectan ni definen solo la vida de estas. Es así como las múltiples y diversas luchas feministas han logrado que el Estado reconozca y se comprometa a garantizar derechos a las mujeres que hasta hace
50 años eran considerados problemas íntimos: el reconocimiento de la violencia física y sexual hacia las mujeres, así como los derechos sexuales y reproductivos, como ámbitos de acción de la política pública de los Estados, son una muestra de ello.

Sin embargo, en América Latina este ámbito de reconocimiento de lo privado se ve bastante limitado por la superposición de otros problemas que tienen como principales sujetas a las mujeres, el principal y más ampliamente reconocido el de la pobreza; asociado a este, está el hecho de que la maternidad es una condición recurrente en las mujeres más pobres, por lo que las políticas orientadas hacia ellas, se diseñan casi exclusivamente en función de este par de condiciones: ser madres y ser pobres. Por otro lado está el hecho de que la última década, los procesos de cambio político en América Latina, encabezados por gobiernos progresistas, plantean un desafío muy grande para las organizaciones feministas y de mujeres comprometidas con el cambio social, sobre cómo avanzar en las reivindicaciones de las mujeres en contextos de luchas nacionalistas, dado que género, clase y nación son campos de acción que tienden a contradecirse generando tensiones en las que por lo general, las demandas de las mujeres deben subordinarse al interés estatalnacional. (Angeleri, 2012: 233). Esto ha devenido en una práctica de recurrente ocultamiento o bien perpetua postergación de las demandas feministas que implican transformaciones desde una perspectiva estructural.

Una dimensión relevante a partir de la cual se puede evaluar cómo han interactuado en la práctica estas tensiones, es aquella en la que se definen los intereses de las mujeres y las políticas públicas de género que se diseñan para atenderlos. En ese sentido, en el proceso bolivariano, todo lo relacionado con políticas públicas de género suponía de entrada un reto, dado que los principales referentes conocidos en esta materia, eran precisamente los planteados por el modelo neoliberal. Esto es: las políticas focalizadas a nivel local para contener $y$ administrar la pobreza. 
La cuestión se hace más compleja si a este balance le agregamos que el proyecto bolivariano se ha planteado como modelo de Estado el socialismo, lo que implica necesariamente mirar con un lente distinto las formas en las que se definen los intereses de las mujeres, así como el diseño y aplicación de las políticas públicas. En este contexto, nuestra propuesta de investigación se orienta a caracterizar la experiencia feminista en el contexto definido como de transición al socialismo, así como las formas en que los intereses de la mujer han sido definidos en el marco de la Revolución Bolivariana desde el Estado y los desarrollos concretos (los contenidos) de la política pública orientada a las mujeres.

\section{Breve sobre la transición}

Un primer asunto que vale la pena aclarar en este punto, es que no entendemos la transición como un proyecto sino como un proceso en el cual ocurren transformaciones sustantivas en el ámbito de la producción y reproducción de la vida; en el cual son superadas paulatinamente las relaciones de dominación entre las personas y a su vez, las relación entre estas y la naturaleza a partir de una política de lo común desde una perspectiva feminista ${ }^{1}$, adaptada a la diversidad de las múltiples subjetividades comprometidas con la transformación social. Siguiendo a Harvey (2011), entendemos la transición socialista hoy, a partir de objetivos mínimos que deben ser comunes, reivindicados y defendidos por todas las fuerzas que impulsan la transformación, a saber:

"(...) respeto a la naturaleza, igualitarismo radical en las relaciones sociales, arreglos institucionales basados, en algún sentido, en el interés y la propiedad común, procedimientos administrativos democráticos (...), procesos de trabajo organizados por procedimientos directos, la vida cotidiana como libre exploración de nuevos tipos de relaciones sociales $y$ acuerdos de convivencia, concepciones mentales enfocadas en la autorrealización en servicio a los demás e innovaciones tecnológicas y organizativas orientadas hacia la búsqueda del bien común en lugar del apoyo al poderío militar, la vigilancia y el egoísmo corporativo ${ }^{2}$. (Harvey, 2010: 10).

Situándonos en la primera década del Siglo XXI, con la emergencia de proyectos nacionalpopulares como el venezolano que ha optado por construir el socialismo del siglo $x \times i^{3}$, han ido quedando rezagadas las discusiones históricas y teóricas sobre lo que el socialismo supone como transición, postergando los debates (y las acciones) orientadas más bien hacia la transformación que es lo que debería ocurrir en dicha transición. Por otra parte está la cuestión de cuál ha de ser el papel del Estado en los procesos de transformación social. De acuerdo con Marx:

"La sociedad actual, es la sociedad capitalista, que existe en todos los países civilizados más o menos libres de aditamentos medievales, más o menos modificadas por las particularidades del desarrollo histórico de cada país, más o menos desarrolladas. Por el contario, el "Estado actual" cambia con las fronteras de cada país (...) El Estado actual es por tanto una ficción. Sin embargo, los distintos Estados de los distintos países tienen en común el que todos ellos se asientan sobre las bases de la moderna sociedad

1 Para la feminista Silvia Federici, entender lo común desde una perspectiva feminista debe empezar por el reconocimiento de que "(...) la "puesta en común" de los medios materiales de reproducción de la vida es el mecanismo primordial a través del cual se crean el interés colectivo y lazos de apoyo mutuo".

2 Harvey también plantea la necesidad de comprender que “(...) El fracaso de esfuerzos anteriores para construir un socialismo y comunismo duraderos debe ser evitado y las lecciones de esa historia, enormemente complicada, deben ser aprendidas. Sin embargo, también debe ser reconocida la necesidad absoluta de un movimiento revolucionario anticapitalista coherente. El objetivo fundamental de dicho movimiento social es asumir el mando tanto de la producción como de la distribución de excedentes".

3 Socialismo del Siglo XXI, es un concepto acuñado en el contexto de la experiencia venezolana para referirse a la construcción de un modelo político de gobierno que supere las desviaciones burocráticas y autoritarias de los socialismos del siglo xx o socialismos reales. 
burguesa, aunque esta se halle en unos sitios más desarrollada que en otros (en el sentido capitalista). Tienen también ciertos caracteres esenciales comunes (...) Cabe entonces preguntarse, ¿Qué transformación sufrirá la estructura del Estado en la sociedad comunista?, o en otros términos ¿qué funciones análogas a las actuales funciones del Estado subsistirán entonces? Esta pregunta solo puede contestarse científicamente y por más que acoplemos de mil maneras la palabra "pueblo" y la palabra "Estado" no nos acercaremos ni un pelo a la solución del problema" (Marx, 2000: 32).

Hay dos aportes fundamentales en este planteamiento de Marx sobre el Estado y el papel que este ha de tener en la nueva sociedad, por un lado que no puede partirse de una definición unilateral del mismo, dado que las características de este cambia según las fronteras y el desarrollo histórico de cada nación y por otro lado, no niega de entrada la posibilidad de que este exista en la sociedad comunista, sino que más bien se plantea la posibilidad de que algunas de sus funciones subsistan en esta nueva sociedad. Un elemento importante de este fragmento, es la alerta en que la identificación entre Estado y pueblo (podríamos decir, el Estado del Pueblo) no le aporta soluciones concretas al problema de la transformación.

Estas preguntas sobre el papel del Estado siguen vigentes hoy, cuando la posibilidad de construir el socialismo en el siglo XXI, se define principalmente por no repetir los errores ampliamente documentados en las experiencias socialistas reales del siglo XX. Uno de los señalamientos más contundentes en el balance de estos procesos, es precisamente la centralización de la política y de todos los órdenes de la vida pública en el Estado, y su poca voluntad política para impulsar transformaciones más allá del control de los medios de producción.

Es en este contexto que el feminismo experimenta tensiones y rupturas con respecto al modelo socialista; una vez conquistado el poder las demandas más radicales de las mujeres por igualdad de oportunidades y garantía de condiciones para el desarrollo de sus capacidades, las mismas son constantemente repriorizadas y muy a menudo postergadas.

\section{Feminismo y socialismo: una relación compleja}

El feminismo se inaugura a finales del siglo XVIII, en el marco de una revolución que proclamaba a la mujer como ciudadana, como sujeto político con la capacidad de hacer y decidir en tanto la sociedad y el Estado garantizaran igualdad de condiciones y oportunidades para ellas. La lucha de las mujeres por politizar temas como el trabajo doméstico, las relaciones sexuales y los derechos reproductivos, han colocado en el debate público que estos no son asuntos privados sino de interés público.

Así, el feminismo ha contribuido de manera importante al interés por la ciudadanía y la democracia, a través de su cuestionamiento a las premisas del liberalismo y la democracia, las analistas feministas revelaron la desigualdad en el centro mismo de los principios universales de igualdad, universalismo e imparcialidad (Molyneux, 2000).

Desde mediados del siglo XIX, hasta la fecha, la vinculación entre los proyectos revolucionarios (anarquistas, socialistas, comunistas, etc.) ha incorporado a sus programas delucha (y enalgunos casos de gobierno) las demandas de las mujeres de garantías plenas a sus derechos en ámbitos señalados como privados y que solo en contextos de transformación social (revolucionarios) han podido ser socializados. Sin embargo, habría que reconocer también que muchas revoluciones no plantearon el problema del estatuto de la mujer de forma significativa o en sí mismo; aún así, es poco probable que se pueda hablar hoy de un proyecto revolucionario que no incorpore en sus programas de lucha y de gobierno los problemas y demandas de las mujeres.

La emergencia de procesos emancipatorios supone la necesidad de incorporar las experiencias de las mujeres y la visión feminista del derecho a ser ciudadanas. El principio autoincentivador y expansivo de la revolución exige la transformación de todas las formas 
de sometimiento de las relaciones sociales y también un sentido trascendental de los procesos culturales alternativos para la construcción de sociedades sin explotación, con equidad e igualdad (Carosio, 2010).

En ese sentido, la historia de las revoluciones socialistas y el feminismo comprometido con la transformación social, se han interpelado mutuamente:

¿Es posible la igualdad real de las mujeres como objetivo último del feminismo sin una transformación social radical? ¿Es posible una transformación social radicalmente emancipatorio sin la igualdad efectiva de las mujeres que propugna el feminismo?

La igualdad en estos términos no es entendida solo como igualdad entre mujeres y hombres, ya que se reconoce que esta no existe de hecho entre los hombres, mucho menos entre las mismas mujeres. La igualdad es entendida ante todo como igualdad de condiciones para el desarrollo de la capacidad de cada quien sin que esta se encuentre limitada por razones de género, por lo que una revolución implicaría trascender las concepciones genéricas, binarias sobre las cuales se organiza la sociedad y el Estado asignando el ámbito de lo reproductivo/privado a las mujeres y el ámbito productivo/público a los hombres. Se reconoce que en la realidad estas oposiciones no funcionan de forma esquemática y que de hecho pueden variar y encontrar excepciones a esta división, sin embargo todos los datos referidos a la diferencia salarial, la libertad reproductiva y las cargas de trabajo doméstico hace imperativo la necesidad de reivindicar dicha igualdad de condiciones de forma específica para las mujeres.

Sin embargo, el sentido exacto del feminismo al interior del proyecto bolivariano, no se reduce al problema de una "igualdad efectiva" como en periodos anteriores, ni siquiera al de igualdad de condiciones para el hacer de las mujeres, sino que se inscribe en un proyecto más amplio de transformación social de la totalidad y no sólo de las condiciones de vida de las mujeres en lo particular, que desde hace mucho tiempo reivindica el ámbito de las condiciones en que se reproduce la vida como piedra angular de la transformación social.

Es por ello que la relación entre feminismo y socialismo ha sido siempre una relación tensa, de acusaciones mutuas sobre las capacidades $\mathrm{y}$ la voluntad real que uno $\mathrm{u}$ otro proyecto realmente tienen para emancipar a las mujeres y/o a la sociedad. En América Latina, esta tensión se ve atenuada por el origen mismo de las luchas feministas, por lo cual, ha sido acusado en no pocas oportunidades de ser una ideología extranjera, que poco o nada tiene que ver con las necesidades reales de las mujeres pobres en este lado del mundo. Así, los proyectos socialistas en la región se enfocan especialmente en las mujeres pobres y en el rol y la función que estas ocupan en términos de movilización social de apoyo a estos proyectos. Así se traza un límite muy claro entre las mujeres pobres y las feministas. Sin embargo, prevalece una tendencia muy fuerte a generalizar los intereses de la mujer en función de la maternidad y el cuidado, a pesar de las múltiples diferencias de aspiraciones y capacidades de las propias mujeres entre si.

La diferenciación al interior del feminismo por la cuestión de clase ha estado muy presente en los proyectos revolucionarios del siglo $\mathrm{XX}$, con el transcurrir del tiempo y la cooptación del propio sistema capitalista de algunas demandas y reivindicaciones feministas en términos de derechos, se han hecho no pocos intentos por borrar las diferencias de clase en el discurso, proponiendo que la cuestión de la mujer sea tratada de forma imparcial y sin mayores distinciones (ya no solo de clase, sino también de etnia y de orientación sexual). Así los intereses de la mujer se fijan a partir del género sin establecer diferencias entre los contextos y entre las propias mujeres

\section{Intereses de la mujer}

Si bien es cierto que la relación entre feminismo y socialismo, no debe darse por obvia, en el caso venezolano, individualidades feministas, organizaciones y redes, encuentran en la doctrina socialista, las mejores probabilidades de avanzar en la emancipación de las mujeres. Un siglo 
después de experiencias socialistas reales, el balance no establece una relación directa entre conquistas de derechos y mayor participación de las mujeres y gobiernos socialistas. Desde mediados del siglo $\mathrm{xx}$, gobiernos liberales han tenido que ceder también frente a la movilización de las mujeres por sus derechos. En este sentido, millones de mujeres en el mundo se han visto beneficiadas de una manera $u$ otra de ciertas políticas de gobiernos que sin importar su signo (socialista o liberal) han implementado políticas sociales y de bienestar en favor de ellas, así como han experimentado también ciertas mejoras en sus derechos legales a través del cumplimiento de leyes sobre igualdad de salario y trabajo. Este programa se viene cumpliendo cabalmente en EEUU y en Europa desde los años 70 del siglo XX.

En América Latina, cuyas experiencias revolucionarias ${ }^{4}$, se inscriben en la perspectiva de lo nacional-popular, una de las críticas fundamentales desde la perspectiva feminista a los modelos socialistas, es que se ha hecho poco para desmantelar mecanismos distintos al de la producción, a través de los cuales se reproduce la subordinación de la mujer per se, en la economía y en la sociedad.

¿Significa esto quedespués detodolosintereses de la mujer no han sido adecuadamente representados dentro de las experiencias socialistas? Veamos

Los debates alrededor de los intereses de la mujer, son especialmente relevantes en los procesos de transformación social, sin embargo, una teoría de los intereses que sea útil para analizar la capacidad de las mujeres de luchar por un cambio social y beneficiarse de él, pasa hoy por el reconocimiento de las diferencias realmente existentes entre ellas, en vez de suponer que componen una subjetividad homogénea. El concepto de intereses de la mujer tal y como es usado por los Estados (sea cual sea su signo) es problemático dado que las mujeres se ubican dentro de sus sociedades desde varios lugares en donde se superponen varias opresiones, como por ejemplo la clase y la etnia, en este contexto, los intereses como grupo se construyen de manera compleja y no pocas veces conflictiva. Molyneuax (1986) propone distinguir tres niveles de intereses de la mujer, que se confunden frecuentemente:

1) Intereses de la Mujer; intereses generales en común a todas las mujeres,

2) Los intereses estratégicos de género: se derivan del análisis de la subordinación de la mujer, apuestan por abolir la división sexual del trabajo, reconocimiento y alivio del trabajo doméstico, la maternidad libre y elegida, entro otros.

3) Los intereses prácticos del género: responden por lo general a la percepción de una necesidad inmediata y no suponen metas estratégicas como la emancipación de la mujer o la igualdad entre los géneros (programas orientados a las mujeres relacionados con la maternidad, de abastecimiento doméstico y bienestar público).

Esta distinción permite enfocar mejor el análisis sobre si un Estado está o no actuando en favor de los intereses de las mujeres, es decir si todos o una parte de esos intereses están representados en el Estado. Un Estado puede ganarse el apoyo de las mujeres mediante la satisfacción de sus demandas prácticas inmediatas o ciertos intereses de clase o ambos. Lo puede hacer también, sin que avancen del todo sus intereses estratégicos objetivos. Ahora bien, el solo hecho de que este Estado represente algunos de los intereses más prácticos o de clase de las mujeres, y por ende conserve su apoyo, no es suficiente para afirmar que está comprometido con la emancipación de las mujeres. (Molyneux, 1986: 347).

Las distinciones expuestas anteriormente nos servirán para referirnos ahora a la Revolución Bolivariana y cómo se han formulado en el marco de esta los intereses de la mujer (de género, estratégicos y prácticos) y cómo les ha ido a las mujeres en dicho proceso.

4 Nos referimos específicamente a las experiencias de Cuba, Chile y Nicaragua, sin intención de homogeneizarlas y/o entenderlas como un mismo proceso, sino enmarcándolas en al ámbito de lo nacional-popular. 
El campo que abarca los intereses o problemas de las mujer, ha experimentado múltiples transformaciones durante todo el siglo $X X$ y lo corrido del XXI. Estas transformaciones han ampliado el horizonte, con respecto al feminismo, aceptando que no existe una única y acabada versión de este por un lado, mientras que la misma idea de revolución socialista ha incorporado en un mismo plano de importancia la emancipación de las mujeres.

En este contexto, la categoría intereses de la mujer, se refiere de forma explícita a la forma en que los Estados y gobiernos socialistas elaboran y difunden los intereses de la mujer, así como a la forma en la que esos intereses entran en juego en un proceso definido como de transición y cómo ayudan, a su vez a definirla.

Sin embargo, nos arriesgamos a plantear la hipótesis de que en el período de transición son las mujeres las que deben formular sus intereses, y que dichos intereses son múltiples, y que estos a su vez no están exclusivamente marcados por el género.

\section{El proyecto bolivariano: la política con respecto a la mujer}

Dado quenoes posible desconocer la capacidad que tiene el Estado de dar visibilidad pública a determinadas demandas y reivindicaciones, al generalizarlas a nivel de la sociedad, en el caso de las mujeres puede contribuir a modificar las percepciones sobre su situación de subordinación y las razones por las que se encuentran en ella, sin embargo:

(...) las políticas públicas hacia las mujeres asumidas desde el Estado, si bien son importantes no pueden contener el conjunto de demandas, reivindicaciones, necesidades y propuestas a largo plazo, porque ellas se ubican en una lógica diferente a la lógica estatal/patriarcal y porque su cristalización implicaría transformaciones profundas e irreversibles de la sociedad (Vargas, 1988: 87).

El Estado es a la vez un espacio y un conjunto de procesos que envuelven un juego contradictorio de posiciones, representadas por y en distintos sectores de la burocracia estatal. Las políticas públicas son entonces el resultado de la configuración de las relaciones de fuerza existentes en cada momento, pero en definitiva, son elaboradas, diseñadas y ejecutadas por quienes tienen el poder en el Estado. La burocracia estatal es al mismo tiempo arena de la lucha política -donde alternan diferentes actores representando intereses privados- y actor social con iniciativas, interlocutor de otros sujetos/as y actores, interprete de un conjunto de directrices políticas. Esta simultaneidad de papeles del accionar de la burocracia estatal muestra cómo las políticas no son simples respuestas a problemas determinados sino que son parte constitutiva de los procesos a partir de los cuales se establecen $\mathrm{y}$ se ponen en relación los diferentes sujetos/as sociales (Anzorena, 2013, pp.37-39).

La experiencia venezolana de la última década está atravesada por un conjunto de tensiones y contradicciones alrededor de las formas en que el proyecto bolivariano ha dado respuestas (o no) a las demandas históricas de las organizaciones feministas y de mujeres. Existen varios ámbitos desde los cuales se puede analizar la relación entre feminismo(s)-intereses de las mujeres-Estado y Transición. Para efectos de esta investigación nos concentraremos en caracterizar como se han priorizado los intereses de las mujeres a partir de definición del carácter socialista de la revolución bolivariana ${ }^{5} \mathrm{y}$ cuáles han sido las políticas públicas creadas para dar respuesta a esos intereses.

5 El 30 de Enero de 2005, en el estadio Gigantinho de Porto Alegre, en un discurso ofrecido en el marco de la clausura del Foro Social Mundial realizado en esa ciudad, Hugo Chávez planteó la necesidad de superar al capitalismo en los siguientes términos: "Cada día estoy más convencido, sin ninguna duda en mi mente y como han dicho muchos intelectuales, de que es necesario trascender al capitalismo. Pero el capitalismo no puede trascendido desde dentro del propio capitalismo, sino a través del socialismo, el verdadero socialismo, con igualdad y justicia (...)" El discurso completo puede ser consultado en: http://es.scribd.com/doc/25071477/ CHAVEZ-H-Discursos-2005\#scribd 
En ese sentido, es necesario distinguir entre los derechos formales que confiere el reconocimiento de las mujeres como ciudadanas y lo que Molyneux denomina "ciudadanía realmente existente de las mujeres", esto es, entre los aspectos político-legales y sociales de la ciudadanía dado el reconocimiento implícito de que por una $u$ otra razón muchos derechos formales no se realizan. "Esta brecha entre derechos formales y sustantivos invita al análisis de cómo se vive la ciudadanía en la práctica: en los tribunales, en la organización política, en el hogar, así como a las interpretaciones que tienen diferentes sectores de la población de sus derechos y de los términos de su participación social o exclusión" (Molyneux, 2000: 7).

A partir del año 2005, los discursos, las perspectivas y puestas en acción del gobierno venezolano se proponen en el orden de la transición al socialismo. En este contexto se parte de la premisa de que hablar de transición implica necesariamente dar cuenta del paso de un orden (de las cosas, de las relaciones sociales, de producción, reproducción, etc.) a otro orden completamente distinto; para el tema específico que nos ocupa, lo que nos interesa es cómo las organizaciones de mujeres y feministas definen ese otro orden en el contexto de la Venezuela Bolivariana.

La propuesta hecha en las elecciones de 1998 sobre la urgencia de elaborar una nueva Carta Magna agrupa una cantidad representativa de mujeres de todas las organizaciones del país, convencidas de la importancia de participar y contribuir en la construcción de un proyecto de nueva sociedad. Para el caso de las organizaciones feministas y de mujeres que participaron en el proceso constituyente, en ese primer momento se trataba de garantizar que los derechos de las mujeres tuvieran rango constitucional, que la constitución expresara -a través de todo su articulado-el principio de la igualdad de derechos y oportunidades, entre hombres y las mujeres, y que se eliminaran las exclusiones y discriminaciones como expresión y garantía de una verdadera democracia participativa.

Este proceso, supuso la re-articulación de las organizaciones feministas que históricamente habían impulsado la lucha por los derechos de las mujeres, relacionados con la familia, el trabajo y el hogar, pero también con la promoción y desarrollo de nuevas expresiones organizativas de mujeres con expectativas para la participación política y de gobierno. En el marco de esta dinámica también fue posible visibilizar la emergencia masiva en el espacio público de los sectores populares del país, al interior de los cuales las mujeres representan un porcentaje alto e importante, y registran altos niveles de participación a nivel comunitario.

Este ejercicio de amplia participación y concreción de propuestas re-configuraría en buena medida las formas de organización y acción social colectiva de las organizaciones feministas y colectivos de mujeres, comprometidas antes durante y después del proceso constituyente con las reivindicaciones feministas, así como también abriría las compuertas de la participación a miles de mujeres habitantes de sectores populares, que en alguna medida las iría acercando a ciertas ideas y prácticas feministas en materia de derechos y participación política. En este primer momento la política institucional se concentró en la premisa de la inclusión y la asistencia, para reducir los niveles de extrema pobreza en los que se encontraba entonces el país. Las políticas sociales de los primeros años de gobierno bolivariano estuvieron concentradas en políticas focalizadas dirigidas a los sectores sociales más pobres mediante las garantías en el acceso a la alimentación, la salud y la educación.

El impacto que este tipo de políticas tiene sobre las mujeres es muy grande, dado que a través de ellas, se atiende en un mismo movimiento a sus hijos y cualquier otra persona a su cuidado, se asigna una gran carga emotiva al compromiso de las mujeres más pobres con el proyecto bolivariano. Esta apelación emotiva de las mujeres como defensoras del proceso, ha sido un mecanismo eficaz para posicionarlas como las principales operadoras (de muy bajo costo) de las políticas sociales y de género desarrolladas una vez superado el período constituyente.

En Venezuela, al igual que la mayoría de los países de la región, la concepción de políticas públicas de género había estado centrada en el 
desarrollo de programas de asistencia dirigidos a proteger la reproducción y la maternidad. A partir de los debates realizados en la Primera Conferencia Mundial de las Naciones Unidas $^{6}$ sobre la Mujer, comienzan a generarse reflexiones impulsadas por mujeres académicas, intelectuales, artistas y colectivos feministas y de mujeres de la región y del sur global, sobre la forma en que este tipo de programas reforzaba la condición de subordinación de las mujeres al hogar, la familia y la maternidad.

No se trata de descartar las políticas asistenciales sino de analizar la forma en la que estas se orientan hacia las mujeres de forma tal que limitan el procesos de ciudadanización de las mujeres, en la medida en que refuerzan el rol doméstico, los estereotipos y las relaciones desiguales de género (Maffía, XIV, en Anzorena, 2013).

En este contexto, y en medio de la creciente polarización política del país, empiezan a manifestarse una serie de tensiones al interior de las organizaciones feministas y de mujeres que interpelaban al Estado al plantear que era posible atender de forma simultánea los problemas de pobreza e inclusión de las mujeres y los problemas estructurales que desde el mismo Estado, y el ordenamiento jurídico limitaban la participación y el ejercicio de ciudadanía plena de las mujeres.

Estas tensiones dan cuenta de una discusión que aún no ha sido lo suficientemente dada en Venezuela sobre lo que Fraser llama el dilema entrelaredistribucióny el reconocimiento(Fraser, 2005). Si bien está ampliamente reconocida la necesidad de políticas de redistribución y asistencia, en un horizonte de transformación social, lo que debe plantearse es la superación de las mismas, por políticas que al redistribuir privilegien el reconocimiento de subjetividades diversas tradicionalmente oprimidas, así como las garantías y condiciones para la participación. La condición de exclusión no se supera solo con mecanismos de redistribución, que en el caso de las y los más pobres, está condicionado por transferencias dinerarias puntuales y en tiempos determinados.

Sin negar la necesidad de las políticas de redistributivas, el reto radica más bien en cómo orientar estas hacia la transformación. Para el caso de las demandas feministas en América Latina, el elemento de tensión de este proceso viene dado por la dificultad de construir autonomía en el contexto de procesos de liberación nacional, para promover políticas que alteren el orden del discurso tradicional sobre las mujeres, como sujetas necesitadas de permanente asistencia, cuidado y protección. Si, la asistencia, el cuidado y la protección son necesarias, pero también es imperativo que existan garantías para la participación en la vida social en igualdad de condiciones, así como la posibilidad de incidir (y decidir) sobre los asuntos relevantes para la nación y no sólo aquellos etiquetados como "de mujeres".

La política del Estado venezolano por redistribuir la renta petrolera en función de programas sociales ha colocado a las mujeres en la posición de ser destinatarias de políticas de género y políticas sociales al mismo tiempo, lo que trae como consecuencia que en términos de indicadores de inclusión pueda verse muy favorecida su situación, mientras que en términos reales de transformación de relaciones de género es poco lo que se avanza. Esto nos ha llevado a plantearnos la interrogante de si en Venezuela la última década ha habido políticas de género o si más bien, ha habido políticas sociales orientadas desde un principio a las mujeres madres más pobres, con la conciencia de que al impactar a las mujeres de forma directa, se impacta por extensión a sus hijos/as y a la familia en general. Es de esta forma que el Estado interviene en la vida de las mujeres, especialmente de las madres pobres o bien las mujeres pobres que tienen la potencialidad de ser madres. Lo que resulta paradójico es que aunque estas mujeres resultan destinatarias de políticas de reconocimiento y beneficiarias de políticas sociales, se presentan como ámbitos escindidos.

6 La primera conferencia mundial sobre la condición jurídica y social de la mujer se convocó en México D.F. para que coincidiera con el Año Internacional de la Mujer, en 1975, observado para recordar a la comunidad internacional que la discriminación contra la mujer seguía siendo un problema en buena parte del mundo. 
Así mismo, vale la pena plantearse la relación que existe en el caso venezolano entre intervención estatal y reproducción/ transformación de las relaciones de género. Hay al menos dos indicadores que nos permiten dar cuenta de una transformación con respecto al período anterior. Por un lado, desde los inicios del proyecto bolivariano han sido mujeres feministas, militantes de izquierda en su mayoría las responsables de conducir e implementar la política pública para las mujeres. Incluso, la institucionalidad de género se reivindica feminista, planteándose superar así la supuesta neutralidad valorativa de la categoría género; por otro lado la incorporación masiva de mujeres provenientes de sectores populares a través de las políticas sociales ha permeado en ellas cierta politización con respecto a su condición de subordinación en la sociedad. Sin embargo esta "masiva" participación de las mujeres debe ser examinada con detenimiento.

Una vez aprobada la nueva constitución en el año 99 se crean dos nuevas instituciones destinadas a atender los problemas y necesidades de las mujeres: el Instituto Nacional de la Mujer

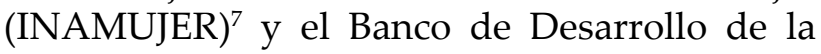
Mujer (BANMUJER), la dirección de cada institución fue asumida por mujeres vinculadas a la izquierda política y con amplias trayectorias en la lucha por los derechos de las mujeres. Sin embargo, esta participación, ha estado condicionada a la convocatoria para el acceso a subsidios o bien pequeños financiamientos para emprendimiento de proyectos productivos. Las dos políticas más relevantes en ese sentido han sido impulsadas por el Banco de Desarrollo de la Mujer (2001-2015) y por la Misión Madres del Barrio (2006-presente). En ambos programas las mujeres pobres son población objetivo $\mathrm{y}$, a la vez, ejecutoras directas de proyectos y subsidios que benefician a los hogares en su totalidad.

Así la participación de las mujeres es definida desde el Estado, acotando los términos y los recursos con los que las mujeres pobres pueden participar y excluyendo de hecho como sujetas de la política al resto de las mujeres que no se inscribe en este grupo social, o bien en estado de necesidad material. En este sentido, es necesario reconocer que los intereses prácticos del género, relacionado de forma directa con políticas de subsidio y atención a la maternidad han estado en el centro de los esfuerzos del gobierno bolivariano dirigidos hacia las mujeres.

Sin embargo, cuando se trata de los intereses estratégicos, relacionados más bien con desestructurar la división sexual del trabajo, el reconocimiento pleno y efectivo del trabajo doméstico $^{9}$ y garantías para la maternidad libre y elegida, los avances son más bien pocos. Veamos.

Con la definición del carácter socialista del proyecto bolivariano en el año 2005, el chavismo propuso construir otro tipo de socialismo, en el sentido de superar dogmas y prejuicios que a la larga minaron las bases de apoyo a este tipo de

7 El Instituto Nacional de la Mujer (Inamujer) fue creado el 25 de octubre de 1999, por disposición de la Ley de Igualdad de Oportunidades para la Mujer, para la defensa y materialización de los derechos de la población femenina y el incremento de su participación en las misiones, planes y programas sociales del Estado, como parte de un nuevo estado de derecho para este sector.

8 Banmujer, o Banco de Desarrollo de la Mujer, es un banco público venezolano creado en el año 2001 con la misión de conceder créditos, microcréditos y otros servicios financieros a grupos y cooperativas de mujeres, sobre todo las de las clases socioeconómicas más desposeídas1 para paliar el desempleo femenino existente en el país. Las mujeres se agrupan en unidades o cooperativas de dos a nueve usuarias y luego de cumplir con el protocolo de solicitud, se les otorga un crédito a cancelar en un período de cuatro años, con un $6 \%$ de interés anual. Luego de cancelarlo, pueden pedir otro crédito por un monto mayor. La idea es que las mujeres inicien proyectos por sí mismas, pero también pueden solicitar talleres de formación según la necesidad.

9 La Constitución de la República Bolivariana de Venezuela aprobada en 1.999 reconoce: “(...) el trabajo del hogar como actividad económica que crea valor agregado y produce riqueza y bienestar social. Las amas de casa tienen derecho a la seguridad social de conformidad con la ley" (Artículo 88, CRBV). 
proyectos. La revolución bolivariana representaba una esperanza para las organizaciones feministas y de mujeres, y con los partidarios de la liberación de la mujer en general, porque en este campo, desde el proceso constituyente en el año 1999 se había prometido mucho. El propio presidente Hugo Chávez se pronunció en repetidas oportunidades sobre la importancia y la necesidad de que la revolución bolivariana impulsara la emancipación de las mujeres.

Estas declaraciones generaron mucha expectativa y esperanza en las organizaciones feministas y de mujeres dada la capacidad práctica que tenía el gobierno para movilizar a miles de mujeres y recursos. Sin embargo, con el transcurrir de los años, dichas expectativas se fueron desvaneciendo, generando al mismo tiempo fracturas al interior del movimiento feminista. Siendo que la mayoría de los esfuerzos para demandar transformaciones han intentado hacerse por la vía parlamentaria, esto es por la modificación de leyes o la creación de unas nuevas, el problema real y concreto radica en la capacidad de ejecución y los recursos financieros, técnicos y humanos que se han dispuesto para el desarrollo de una política pública feminista.

A diferencia de otros proyectos revolucionarios, el proyecto bolivariano no tenía un programa específico para llevar a cabo esta emancipación de la mujer con la que decía estar comprometido. Además la diversidad ideológica al interior del propio chavismo ha generado constantes tensiones sobre cuál es el papel de la mujer y que derechos deben garantizársele.

A la luz del balance de la última década, la brecha entre la promesa de emancipación hecha desde la institucionalidad y la realidad es amplia, si tomamos como indicadores fundamentales de emancipación y autonomía de las mujeres los siguientes:
1) El control sobre su cuerpo (autonomía física) La autonomía física se expresa en dos dimensiones que dan cuenta de problemáticas sociales relevantes, fundamentalmente: el respeto a los derechos sexuales y reproductivos de las mujeres y la violencia de género.

En Venezuela, el proceso de transformación social experimentado la última década no ha impactado de forma significativa el imaginario tradicional sobre las mujeres y su papel en la sociedad, es por ello que la maternidad como destino manifiesto tiene una alta valoración social. En ese sentido el Estado se ha limitado a diseñar políticas dirigidas a las mujeres, en tanto madres, por lo que la autonomía física de las mujeres, en el sentido en el que ha sido definida anteriormente presenta serias restricciones en cuanto a su capacidad de decidir sobre su cuerpos. Así, no existen políticas ni programas permanentes de educación sexual en ningún nivel educativo, la tasa de embarazo adolescente es la más alta de Sudamérica, la mortalidad materna es alta y el aborto está penalizado ${ }^{10}$, salvo en los casos en los que peligra la vida de la madre. El actual código penal -vigente desde 1936- violenta el derecho a la soberanía sobre el cuerpo y los derechos sexuales y reproductivos de las mujeres. En este contexto de ilegalidad, las interrupciones del embarazo se realizan en condiciones de riesgo, por lo que se estima que al menos el $13,78 \%$ de la mortalidad materna está relacionada con esta causa. Es importante destacar que quienes mueren por practicarse abortos inseguros son las mujeres más pobres y las adolescentes con embarazos precoces no deseados.

La ilegalidad del aborto, transforma el derecho de las mujeres a decidir libremente sobre su maternidad y su propio cuerpo en un acto criminal y riesgoso. Así, el Estado ha desestimado las

10 El Código Penal vigente en Venezuela penaliza el aborto en los siguientes casos: Art. $430^{\circ}$ Aborto Procurado: La mujer que intencionalmente abortare, valiéndose para ello de medios empleados por ella misma, o por un tercero, con su consentimiento, será castigada con prisión de seis meses a dos años. Art. $431^{\circ}$ Aborto provocado o consentido: El que hubiere provocado el aborto de una mujer, con el consentimiento de ésta, será castigado con prisión de doce a treinta meses. Si por consecuencia del aborto y de los medios empleados para efectuarlos, sobreviene la muerte de la mujer, la pena será la de presidio de tres a cinco años; y será de cuatro a seis años, si la muerte sobreviene por haberse valido de medios más peligrosos que los consentidos por ella. 
graves consecuencias biológicas, sociales $\mathrm{y}$ psicológicas que derivan de embarazos no deseados y de penalizar el aborto. Detrás de cada aborto clandestino, existe un trasfondo de irresponsabilidad y abandono a las mujeres por parte de la sociedad y el Estado que es el principal responsable de garantizar la vida y los derechos. Sin embargo en Venezuela ha sido prácticamente imposible, promover esta discusión de forma pública y mucho menos que el Estado la acompañe, al menos no de forma integral y siempre con eufemismos que apelan a la prevención, que tampoco se hace, o bien reformas parciales de código que tienen ya un lustro estancadas en el parlamento dados los fuertes prejuicios -sexistas y religiosos- que abanderan las y los parlamentarios responsables de promover el debate sobre el proyecto.

Mantener la ilegalidad del aborto, expresa la convicción que aún existe en el Estado sobre el deber ser madres de las mujeres sin importar lo que estas quieran ni las condiciones en los que esos embarazos no deseados se producen.

Esta restricción de no poder pensar el cuerpo de las mujeres más allá de la maternidad, es lo que a su vez ubica el embarazo adolescente en Venezuela como el problema más grave en materia de derechos sexuales y reproductivos De acuerdo con un informe elaborado por la Red de Colectivos Feministas La Araña Feminista, para el año 2014:

“El problema más grave en el ejercicio de los derechos sexuales en Venezuela, lo constituye el embarazo adolescente: el $24 \%$ de las nacimientos vivos registrados en el país ocurre en adolescentes entre los 15 y 19 años. De aproximadamente 600.000 embarazos registrados anualmente, más de 120.000 partos corresponden a niñas y jóvenes venezolanas (...) el embarazo adolescente, es mayor en los segmentos más pobres. Las madres adolescentes de hoy, son a su vez hijas de las madres adolescentes de ayer" (Informe Alternativo de la Red de Colectivos La Araña Feminista, 2014:8).
Este dato, sea quizás el punto clave desde el cual explicar las limitaciones que tienen aún hoy las mujeres venezolanas, especialmente las más pobres, para desarrollar la autonomía de sus cuerpos, dado que no es un problema relacionado solo con la salud, sino principalmente educativo y socio-cultural. Esta situación da cuenta de una desatención importante hacia la educación sexual y el ejercicio de los derechos sexuales en condiciones seguras, autónomas y responsables.

En lo que respecta a la Violencia de Género ${ }^{11}$, pese a los avances legislativos, las políticas y programas de prevención a los que obliga la Ley Orgánica por el Derecho de las Mujeres a una vida Libre de Violencia se cumplen de manera fragmentaria y con un alcance limitado. Tal y como ocurre con los derechos sexuales y reproductivos no ha habido una intervención pública permanente que problematice y condene socialmente la violencia contra las mujeres, muy por el contrario, en el sentido común general siguen apareciendo como un problema privado.

2) Capacidad de generar ingresos y recursos propios (autonomía económica) La autonomía económica se explica como la capacidad de las mujeres de generar ingresos y recursos propios a partir del acceso al trabajo remunerado en igualdad de condiciones que los hombres.

En Venezuela, a pesar de la protección al trabajo y la promoción de las mujeres a la economía social a través de la gestión de proyectos productivos en el ámbito comunitario, persisten diferencias importantes por sexo en los ingresos, especialmente en los segmentos con menor nivel educativo, también se mantiene la segregación laboral siendo las ocupaciones "de mujeres" o feminizadas como el magisterio, la enfermería, la costura y el cuidado en general, las peor remuneradas.

Así mismo persiste la cultura que adjudica a las mujeres la totalidad de los trabajos del hogar y el cuidado de los hijos e hijas, personas

11 En el año 2007 se aprueba la Ley Orgánica para el Derecho de las Mujeres a una Vida Libre de Violencia, que reconoce 18 tipos de violencia contra las mujeres. 
con discapacidad y mayores dependientes, de manera que estas obligaciones sexo-genéricas actúan como un limitante importante a la hora de la búsqueda y ocupación laboral, es por ello que es más frecuente encontrar en las mujeres historias laborales discontinuas y menos consolidadas, lo que limita a la larga la capacidad de generar ingresos y recursos propios.

El valor que tienen las labores del cuidado no es considerado en términos del tiempo y la contribución de las mujeres a la economía.

3) Plena participación en la toma de decisiones que afectan su vida y su colectividad (autonomía en la toma de decisiones) La autonomía en la toma de decisiones se refiere a la presencia de las mujeres en los distintos niveles de los poderes del Estado y a las medidas orientadas a promover su participación plena y en igualdad de condiciones en su entorno social.

Si bien podría afirmarse que la participación social de las mujeres ha crecido a través de su incorporación en programas educativos de base y formal, así como en los ámbitos de la organización social y comunitaria, también es cierto que esta participación no se relaciona de forma directa con la capacidad que tienen las mujeres de tomar decisiones en ámbitos que afectan su vida y su comunidad como tal. Es por ello que el trabajo comunitario realizado por las mujeres en Venezuela, también es señalado como una jornada más de trabajo que recae sobre las mujeres. Es decir esta mayor participación, no ha tenido como correlato el reconocimiento de necesidades específicas de las mujeres y mucho menos una distribución equitativa en las tareas del hogar, el cuidado y la crianza de los/as hijos/as.

Este balance y el contexto en el que nacen las políticas sociales y de género en la Venezuela Bolivariana, nos plantea la necesidad de establecer con precisión el fundamento de las mismas, teniendo en cuenta a todos los actores (decisores y beneficiarios) pero también la dinámica política concreta que en el caso venezolano ha supuesto un período continuo de inestabilidades, rupturas $\mathrm{y}$ tensiones entre los actores y en el propio contexto.
A partir del año 2006, es necesario ubicar también las características que estas políticas adquieren en el marco de la apuesta por la construcción del socialismo, esto es:

"La definición que se haga desde del Estado, del momento histórico, de las relaciones de fuerza entre Estado y sujetos/as sociales. Por ello, en algunas coyunturas estas pueden tener como objetivo garantizar los derechos sociales de los/as ciudadanos/as, mientras en otros momentos pueden tomar la forma de políticas asistencialistas, que tienen como objetivo atender alguna situación particular que se considera desventajosa. No es lo mismo una política social o de género en un Estado intervencionista, socialista o en uno neoliberal" (Anzorena, 2013:43).

Así, el análisis de las políticas sociales y de género son un indicador importante para evaluar la correspondencia entre la forma en la que el Estado venezolano se define y las relaciones que las políticas sociales y de género que impulsa, realmente transforma o bien reifica.

El contexto en el que nacen las políticas sociales y de género en la Venezuela Bolivariana, es un contexto marcado por una alta polarización política que plantea de forma constante escenarios de confrontación entre quienes están a favor y/o en contra del gobierno. En ese sentido la forma en la que se han desarrollado las políticas sociales, ha generado intensos debates en torno al asistencialismo y la transformación, en ese sentido es importante tener en cuenta que:

“La Diferencia entre una política con perspectiva de género y una política social dirigida a las mujeres está dada por la forma en que se concibe a la sujeta social destinataria de las acciones. Mientras que una política social asistencialista busca atender situaciones particulares que se consideran desventajosas, una política con perspectiva de género entiende la protección de los derechos de los ciudadanos como responsabilidad ineludible del Estado. Esta última apuntaría a la autonomía y al pleno ejercicio de la ciudadanía, a la promoción de los derechos de las mujeres a fin de equiparar las desigualdades sociales, es decir, distintas de las formas asistencialistas de intervención estatal 
que refuerzan roles y estereotipos. (Staubli, citada por Anzorena, 2013. 43).

$\mathrm{Si}$ bien es cierto que la experiencia venezolana normativa y discursivamente ha propuesto superar la tradición asistencialista, en la práctica las políticas sociales y muy especialmente las políticas dirigidas hacia las mujeres han reforzado este carácter de asistencia y dependencia de las mujeres frente al Estado, no solo desde una perspectiva económica sino incluso desde una perspectiva afectiva. La relación de las mujeres de sectores populares beneficiarias de políticas sociales con el presidente Chávez por ejemplo, estaba marcada por el sentimiento paternal de protección en el que las mujeres se sentían reivindicadas y reconocidas. Esto no sería necesariamente un problema si esta relación no reforzara la necesidad de las mujeres de un hombre (que en este caso encarnaba al Estado) que las proteja.

SindesconocerqueVenezuelahaexperimentado transformaciones sociales importantes en las formas de acción colectiva, especialmente las comunitarias, esto no necesariamente ha implicado la transformación estructural de relaciones de dominación en la medida en que esta explosión de organización comunitaria precisamente ha reforzado los estereotipos sobre las mujeres-madres y su vocación "natural" por la administración y el cuidado, tal y como está expresado en el último Plan para la Igualdad y la Equidad de Género 2013-2019:

“(...) Este plan lleva por nombre Mamá Rosa, mujer venezolana quien sintetiza en su significación histórica el hermoso papel que juega la mujer madre, la mujer abuela, la mujer patriota en la creación del hombre y la mujer nueva que consolidará la alborada de un mundo mejor".

Como indica Maxine Molyneux (2003), el problema con este enfoque es que el reconocimiento del aporte social de las tareas del cuidado de las mujeres ha contribuido a crear una serie de expectativas sobre su papel en los proyectos de desarrollo que ha tenido efectos perversos para ellas. Por una parte, se considera que las mujeres están naturalmente predispuestas a servir a sus familias y a sus comunidades, por ser menos individualistas y por la forma en la que están incorporadas en los vínculos familiares y vecinales como consecuencia de sus responsabilidades en el ámbito de la reproducción social. Esto hace que se termine considerando todo lo relacionado con lo comunitario, la salud y la educación de la familia, hasta la protección del medio ambiente como terreno de las mujeres. Esto tiene un elevado costo no reconocido para ellas, porque por una parte se aprovecha su trabajo voluntario impago para desarrollar los proyectos participativos que desde organismos nacionales e internacionales se promueven, y por otra se considera que el capital social, esto es, las estrategias de supervivencia y las formas de cooperación que las mujeres portan y aportan (hacen que América Latina sea menos pobre en las mediciones) es la panacea para gestionar la pobreza, como si por si solo este capital pudiera suplir los recursos y el diseño de las políticas públicas que realmente transformen el estado de subordinación de las mujeres, especialmente de las más pobres. (Molyneaux, 2003, p 334-338).

\section{4) Participacion de las mujeres en la venezuela bolivariana: movilización del trabajo afectivo ${ }^{12}$}

12 El Trabajo Afectivo como concepto se utiliza hoy para describir las nuevas tareas y trabajos desarrollados en el sector servicios o para conceptualizar la naturaleza del trabajo en la era pos-fordista: para otros supone un sinónimo de trabajo reproductivo y un punto de partida para replantear y reestructurar las bases del discurso feminista sobre la transformación. Para otros autores como Hardt y Negri, "el trabajo afectivo es el que produce o manipula afectos como las sensaciones gratas o de bienestar, la satisfacción, la excitación o la pasión". Silvia Federici, elabora una crítica a ambas definiciones del Trabajo Afectivo, en la medida en que al ubicarlo en el sector servicios, es desgenerizado ocultando así la gran carga con la que este opera de forma específica sobre las mujeres. 
"Bajo la hegemonía del Trabajo Inmaterial, la personalidad y la subjetividad de los trabajadores tienen que estar preparadas para organizarse y recibir órdenes"

Mauricio Lazzarato

"Los gobiernos revolucionarios tienden a ver la importancia de reforzar la posición de la mujer en el primer período de transformación económica y social, en términos de su contribución al cumplimiento de por lomenos tres metas: extender la base política de apoyo al gobierno, incrementar el tamaño o la calidad de la fuerza de trabajo activa y ayudar a vincular con mayor firmeza a la familia con el proceso de reproducción social. La primera meta, extender o mantener la base del poder del Estado busca atraer a las mujeres a las nuevas organizaciones políticas tales como las de mujeres, juventud, sindicatos, el partido y muy especialmente a las asociaciones de barrio. Se expresa frecuentemente el temor de que si no se politizan las mujeres podrían no cooperar con el proceso de transformación social ${ }^{13}$. En general, la movilización política de las mujeres supone un esfuerzo importante para persuadirlas de que el Estado representa sus intereses, así como las preocupaciones más universales (nacionales, humanitarias, etc).

La forma más efectiva de movilizar a las mujeres pasa tradicionalmente por exaltar su papel en el nacimiento y consolidación de la nación, especialmente su aporte como madres y portadoras de lo bueno por naturaleza. Reivindicar el valor de las mujeres en estos términos implica movilizar al mismo tiempo afectos y pasiones que son fundamentales para concretar el apoyo o rechazo a un proyecto político determinado. En el caso venezolano, desde muy temprano el proyecto bolivariano incorporó en sus discursos el hablarle de forma directa a las mujeres más pobres, reconociendo su situación de exclusión y el compromiso con sacarlas de la misma para ser incorporadas de forma plena a la reconstrucción nacional. En este sentido, nos resulta apropiado hacer uso de la categoría de Trabajo Afectivo para dar cuenta de la dinámica y los efectos reales de la participación de las mujeres en la Venezuela Bolivariana.

En los estudios feministas el concepto de Trabajo Afectivo, proporciona una herramienta de análisis, para explorar nuevas formas de explotación laboral (mayoritariamente femeninas) así como para analizar los nuevos modos de subjetividad y proyección, estimulando la investigación empírica sobre los cambios sufridos por el trabajo reproductivo y sus sujetos al entrar en la esfera públicomercantil. El trabajo de las mujeres, remunerado o no, dentro o fuera de la casa puede describirse como el mayor espacio común de trabajo afectivo del planeta. De acuerdo con Silvia Federici:

“Las mujeres son los sujetos centrales del trabajo emocional (...) aunque este sea un trabajo asalariado y de atención al público, en esencia se trata del mismo tipo de trabajo que ellas han realizado siempre (...) ante la falta de otro tipo de recursos y dependiendo del hombre para la obtención de dinero, la mujer siempre ha transformado sus emociones en valores (activos), ofreciéndoselos al hombre como contraprestación por los recursos materiales de los que ella carecía (...) el aumento del sector servicios incrementa la sistematización, la estandarización y la producción en masa del trabajo emocional, pero su existencia radica aún en el hecho que, la mujer desde la infancia fue entrenada para una relación instrumental con sus emociones. (Federici, 2013:58).

En este sentido, las formas de promover la participación de las mujeres está más bien relacionada con una visión esencialista que sostiene que estas tienen una suerte de tendencia natural al comunitarismo porque están menos motivadas por un individualismo egoísta, dado que su papel en la familia y su responsabilidad de proveedoras las predispone al activismo

13 Las mujeres son consideradas como potencialmente-y de hecho- más conservadoras que el hombre dado su lugar dentro de la división social del trabajo. Más estratégicamente suelen ser consideradas también como agentes decisivos del cambio revolucionario, dado que su radicalización desafía antiguas costumbres y privilegios dentro de la familia y tiene efectos importantes sobre la próxima generación a través de la influencia que estas ejercen sobre sus hijos. 
popular y al trabajo comunitario. En América Latina, esta forma de entender la participación de las mujeres ha sido una constante en los procesos de transformación social: en tanto que comunitarias naturales, se deposita en las mujeres el trabajo voluntario como parte activa en la política de base popular.

Desde una perspectiva feminista, esta forma de promover la participación de las mujeres es problemática, en tanto que: por un lado no atiende los intereses estratégicos de las mujeres y no es definida orgánicamente por quienes participan. En este caso, es desde el Estado que se definen los intereses de la mujer y el espectro de libertad en que puede perseguirlos. La mujer como militante de base es a la que se la deja actuar, pero se la encasilla como trabajadora afectiva definida por sus funciones en la esfera privada abuela, madre tía o simplemente servidora de su comunidad. Así todo lo que entra en la esfera de lo "popular" es decir del activismo de base está definido por la lógica de lo participativo sin que esto necesariamente transforme relaciones de dominación, sino que más bien se aprovecha de ellas, en nombre de la autogestión y el comunitarismo:

“(...) más que una forma autónoma, autoorganizada, espontanea, productora de formas elementales de comunismo, para los trabajadores del Trabajo Afectivo, se trata de una experiencia mecánica, alienante, realizada bajo el mandato directo en el que son espiados y realmente medidos y cuantificados respecto a su capacidad para producir valor, tanto como en cualquier otra forma de trabajo físico. También se trata de una forma de trabajo que genera sentimientos más intensos de responsabilidad y ocasionalmente incluso de orgullo en los trabajadores, minando así cualquier potencial de rebelión contra la injusticia sufrida" (Federici, 213:63).

Así el problema de la mujer es menos su lucha contra el machismo y ser reconocida como ciudadana que la forma como el Estado -y otros poderes- "formatean" una y otra vez su libertad de acción estableciendo los límites de lo que pueden reivindicar en términos de participación.
Mientras tanto, las mujeres están todavía sujetas a relaciones que quiere someterla a cierto tipo de servidumbre, la mujer-funcionaria que le da el formato de lo que puede querer o como puede actuar, y el Estado a quien le conviene que trabaje gratis para siempre, y también a los poderes semióticos que se meten en su deseo generando expectativas $y$ frustraciones con respecto a sus propios cuerpos.

“(...) Ni el trabajo afectivo, ni la producción biopolítica pueden responder hoy a los asuntos clave de las vidas de las mujeres, a saber: la crisis que las mujeres enfrentan al tratar de reconciliar el trabajo remunerado con la reproducción y el hecho de que la reproducción social recae sobre el trabajo no remunerado de la mujer (ya que la misma cantidad de trabajo que se extrae del hogar ha vuelto a él como efecto de los recortes en sanidad y cuidados hospitalario, y de la reducción del pequeño comercio) debido también a la expansión -mundial- del trabajo doméstico, pero sobre todo a la continuidad del hogar como un imán que atrae el trabajo impago o mal pagado (...) generalizar el trabajo afectivo, esto es, su diseminación sobre cada forma de trabajo, nos lleva a una situación pre-feminista en la que no solo la especificidad sino además la existencia misma del trabajo reproductivo de las mujeres y las luchas que estas llevan a cabo en ese terreno se vuelven invisibles" (Federici, 2013: 60).

Una pregunta clave a la hora de hacer un balance de la experiencia venezolana es hasta qué punto la movilización de las mujeres en función del cuidado y la comunidad ha redundado realmente en una mejor calidad de vida para ellas y sobre todo en la formulación clara y explícita de sus intereses sin que estos estén mediados o contenidos desde el Estado.

¿Qué tipo de libertad se ha producido por y para la mujer en la Venezuela Bolivariana?, ¿de qué forma esa libertad puede ser "consumida" por el Estado o el mercado, por ejemplo pero dentro de un cierto sistema de restricciones?

Si bien es cierto que la constitución del año 1999 amplió de forma excepcional derechos para promover y garantizar la autonomía de 
las mujeres en todos los ámbitos de su vida, con el transcurrir de los años ha sido muy difícil concretarlos, y por el contrario se ha venido reduciendo cada vez más la posibilidad de avanzar en la concreción de intereses estratégicos (los que se esperarían fueran la prioridad en un proyecto de transformación social) y más bien se ha orientado la política pública en la atención de intereses prácticos dirigidos a grupos de mujeres muy específicos (madres y pobres) y según las leyes a todas las mujeres en general.

La maternalización de la mujer por parte del Estado y por otras mujeres, operadoras de la política pública, es la forma más eficaz de mantener inalteradas sus condiciones de sujeción en la medida en que se manifiesta no tanto como coerción externa, sino como impulso innato en cada mujer, dándole al Estado la oportunidad de operar sobre ese sentimiento, sobre ese afecto de las madres, y explotar el mismo en función de su proyecto.

Entre la mujer pobre (destinataria de la política de participación) que aporta trabajo afectivo, que organiza actividades en su comunidad y la mujer funcionaria o la académica, de clase media, existe una distancia en términos territoriales, culturales y simbólicos que restringen la posibilidad de avanzar en la formulación de intereses estratégicos específicamente relacionados con la experiencia venezolana. Un ejemplo de esta limitación se expresa en la forma en que quedaron planteados los "retos" de la mujer venezolana, en el último Congreso Venezolano de las Mujeres, convocado desde el Estado:

"Las mujeres patriotas y revolucionarias están comprometidas con defender, preservary profundizar el legado del Comandante Eterno, Hugo Chávez Frías. En este sentido, apoyamos de forma irrestricta al compañero Presidente Obrero Nicolás Maduro Moros, frente a cualquier escenario y en cualquier circunstancia" (Programa de Lucha de las Mujeres Patriotas y Revolucionarias. Documento Base para el Debate)

Este documento estaba ya de hecho formulado antes de la realización del Congreso y solo fue distribuido para su validación y apoyo irrestricto, el problema con este tipo de compromisos es que los problemas específicos de las mujeres se diluyen en el proyecto nacional y pasan a un segundo plano. Así mismo apelar al patriotismo, al compromiso y a la defensa del legado del presidente Chávez función como un dispositivo para movilizar el trabajo afectivo de miles de mujeres vinculadas con el Estado.

Sin embargo esta es una comprensión distorsionada de lo que el trabajo afectivo supone en función de la transformación social. Siguiendo a Federici,

\begin{abstract}
“(...) solo cuando consideramos el trabajo afectivo como reproductivo en su doble y contradictoria función, como la reproducción de los seres humanos y simultáneamente como la reproducción de la fuerza de trabajo, podemos imaginar y plantear distintas maneras y formas de lucha y rechazo, que fortalezcan a los que cuidamos en vez de destruirlos. La lección dada por el movimiento feminista ha sido crucial a este respecto, ya que se reconoce que el rechazo de la mujer a la explotación y el chantaje emocional, que se encuentran tanto en el núcleo del trabajo doméstico no remunerado como en el trabajo de cuidados no remunerados, libera a su vez a aquellos que dependen de este trabajo" (Federici, 2013: 62).
\end{abstract}

Así el Trabajo Afectivo que se analiza desde el feminismo no tiene nada que ver con la emancipación, muy por el contrario explota aún más la situación de subordinación de las mujeres en nuestra sociedad, y limita su "participación" a asuntos relacionados con el cuidado, visto esto como una extensión del trabajo que ya realiza en el hogar, que además de impago o mal pagado, no tiene ningún valor social, por lo que exaltarlo es aprovecharse del mismo.

Asílaparticipacióndemilesdemujeresvenezolanas en procesos de organización comunitaria está más relacionada con la gestión del espacio público como una extensión del hogar que con las necesidades específicas que tienen las mujeres al interior de sus hogares pero también en el propio espacio público. Por lo que el saldo al final de cuentas son miles de mujeres agotadas y con los mismos problemas de hace una década. 
5) Intereses y justicia de género: una tarea aún pendiente

A la luz del contexto venezolano hoy se hace necesario dar un paso más y preguntarse acerca de la efectividad de las políticas que tienen como destinatarias a las mujeres en relación con sus objetivos de justicia de género. Para definir una política como justa desde el punto de vista del género, es necesario considerar el qué y el quién de la justicia. En el caso del género, las demandas de justicia, no se hacen desde un solo lugar, y en este sentido el aporte de Nancy Fraser (2008) es central.

El género no es una clase social ni un estatus, las injusticias de género no pueden ser atribuidas solamente a un factor, por consiguiente la articulación de demandas de género surge tanto desde las injusticias económicas como de las de reconocimiento y, también desde la forma en que se organiza la acción política y la toma de decisiones. La propuesta de Fraser permite analizar tres dimensiones del orden social actual -que si bien pueden ser separadas analíticamente, están interrelacionadas entre sí- dimensiones en las que la justicia debe operar: la justicia redistributiva, la justicia de reconocimiento y la de participación y representación política.

Esta separación analítica permite ver las tensiones especialmente entre las soluciones a las demandas redistributivas y las soluciones a las demandas de reconocimiento. Las demandas de redistribución despojan a los grupos de sus diferencias específicas, apuntan a la derogación de las diferencias que implican desigualdades en la distribución, de modo que promueve la igualdad de los grupos. Las demandas de reconocimiento ponen el foco en la especificidad de un grupo y la afirmación de su valor, tiende así a la diferenciación de los grupos.

En el análisis de las políticas se debe considerar esta aparente contradicción de objetivos, que lleva a que las exigencias estén en tensión e incluso aparentemente se interfieran. Una política justa debiera poder entrelazar de manera mutuamente positiva objetivos redistributivos con objetivos de reconocimiento y objetivos de representación. Debe, entonces, apuntar en sus fundamentos a defender una política social de la igualdad y a una política cultural de la diferencia (OIG-CEPAL, 2012: 4).

Si bien es cierto que miles de mujeres se han visto beneficiadas con las políticas redistributivas del gobierno bolivariano desde el año 99. Las estructuras fundamentales de la desigualdad no han sido desmanteladas y de alguna forma han sido reforzadas por el estado al colocar a las mujeres como sujetas necesitadas de permanente asistencia. Las políticas sociales tenían como objetivo beneficiar a los más pobres y satisfacer necesidades básicas de salud, vivienda, educación, alimentación. Sin embargo también es un hecho que mientras las mujeres pobres se beneficiaron de la política de bienestar, han sido al mismo tiempo las más vulnerables a las restricciones económicas y especialmente a la escasez de productos básicos.

En términos de los intereses prácticos femeninos, estas políticas de redistribución también tuvieron efectos de género y de clase. Dado que su lugar en la división social del trabajo se mantiene inalterado, las mujeres siguen siendo desproporcionalmente responsables del cuidado de los niños y de la salud familiar por lo que su interés gira de manera particular alrededor de necesidades muy específicas como la vivienda y la comida.

La relación entre socialismo y feminismo plantea reservas importantes sobre el la posibilidad de avanzar en la emancipación de las mujeres, aún en un proyecto que reivindique un programa de liberación para las mismas. Siguiendo a Molyneux:

“En cuanto a los intereses estratégicos del género, a pesar de ser reconocidos en el discurso y en el programa oficial para la emancipación de la mujer, siguen definiéndose de manera bastante estrecha ya que se basan principalmente en criterios económicos. Las teorías feministas de la opresión, la crítica a la familia y la dominación masculina tienen poco impacto sobre el pensamiento oficial y de hecho se suprimen a veces por considerársele "demasiado radicales" y una amenaza para la 
solidaridad en el tejido popular" (Molyneux, 1985: 358-359).

En el caso venezolano, la política pública que debería atender todos los intereses de las mujeres, incluyendo los formulados por ellas mismas, se dirigen de forma casi exclusiva a las mujeres-madres. Una oleada de apelación a la maternidad invade el espacio público, probablemente como efecto de la herencia de tradiciones políticas más extendidas. Los programas sociales ponen en escena la cuestión de la extensión social del rol maternal. La Misión Madres del Barrio está dirigida a las mujeres, pero busca impactar en las personas que ellas tienen a su cargo, mientras se espera que esos beneficios se extiendan a sus hogares y a la comunidad. Las mujeres madres como administradoras son una garantía para llegar a todos/as los/ as miembros de la familia. A partir de ellas se puede asistir toda una gama de problemas relacionados con el cuidado. No es de extrañar entonces que el cuidado de los/as niños/as sea el trabajo más difícil de eludir para las mujeres y el que le reporta más beneficios a cualquier Estado (capitalista o socialista), sobre todo en contextos de crisis y desempleo, donde se considera que liberar a las mujeres de la esfera doméstica empeora la presión sobre un mercado laboral que no ofrece lugar para todos, menos para todas (Molyneux, citada por Anzorena, 2013: 226227).

No es posible resolver hoy la cuestión de los intereses del género y sus medios de representación sino se discute la forma de Estado, las políticas públicas y los programas apropiados para transitar al socialismo, por lo tanto no es solo una cuestión de si el Estado representa o no los intereses de las mujeres, sino de cómo y hasta qué punto los representa.

Dado que los intereses son representados a través de lo que el Estado hace (y también lo que no) las políticas sociales serán siempre un buen indicador del compromiso de un determinado proyecto político en el poder con la transformación social, y con la emancipación de las mujeres en particular.
ENTORNOS, Vol. 29, No. 2, Noviembre 2016

\section{Referencias}

Angeleri, Sandra: LaArañaFeminista: Estableciendo los Parámetros para el Estudio de una Experiencia de Articulación Feminista y Socialista. En Revista Venezolana de Estudios de la Mujer. Vol. 17 № 39. Pp. 225-234. Centro de Estudios de la Mujer, UCV, Caracas, 2012.

Anzorena, Claudia: Mujeres en la trama del Estado: una lectura feminista de las políticas públicas. (Mendoza -1a ed.-. Ediunc, 2013).

Bambirra, Vania: La Mujer Chilena en la Transición al Socialismo, DocumentosSuplemento de la Edición № 133 de Punto Final. Martes 22 de Junio de 1971. Santiago, Chile.

Carosio, A y Vargas, I: Feminismo y Socialismo. Fundación Editorial El Perro y la Rana, Caracas, 2010.

Eisenstein, Zillah: Lo Público de las Mujeres y la Búsqueda de Nuevas Democracias. Documento entregado en la "Conferencia Mujer y Ciudadanía", Londres, julio de 1996.

Federici, Silvia: Sobre el Trabajo Afectivo, en La Inacabada Revolución Feminista. Mujeres, reproducción social y lucha por lo común. $1^{\text {a }}$ Edición, México 2013. Ediciones desde Abajo.

Fraser, Nancy,: “¿De la Redistribución al Reconocimiento? Dilemas en torno a la justicia en una época "postsocialista" en Iustitia Interrupta: Reflexiones Críticas desde la posición "post-socialista", Capítulo I, (Santa Fe de Bogotá, Siglo de Hombres Editores, 1997).

Harvey, David: Organizarse para la Transición Anticapitalista, en http://www.rebelion. org/noticia.php?id=165505

Informe Alternativo de la Red de Colectivos La Araña Feminista, Caracas 2014. 
Kollontai, Alexandra: Los fundamentos sociales de la Cuestión Femenina (extractos): Tomado de la edición digital de Alexandra Kollontai: Los fundamentos sociales de la cuestión femenina y otros escritos, Tamara Ruiz (ed.). En Lucha: España, 2011. http:// www.enlucha.org/site/?q=node/15895

Lazzarato M, Negri, A: Trabajo Inmaterial. Formas de Vida y Producción de Subjetividad. DP\&A Editora, Río de Janeiro, 2001.

Molyneaux, Maxine: ¿Movilización sin Emancipación?: Intereses de la Mujer, el Estado y la Revolución: El caso de Nicaragua. En: Coraggio, José Luis y Deere, Carmen Diana (Coords) La Transición Díficil: La autodeterminación de los pequeños países periféricos. Siglo XXI Editores.

Molyneaux, Maxine: Género y Ciudadanía en América Latina, en Women's Movements in International Perspective: Latin America and Beyond, Cap. 7. Palgrave, Nueva York, Londres, 2000.

Molyneux, Maxine, Movimientos de Mujeres en América Latina. Un estudio teórico comparado. (Madrid, Cátedra) 2003.

Observatorio de Igualdad de Género de América Latina y el Caribe http://www.cepal.org/ oig/

Observatorio Bolivariano de Género: Políticas Públicas Dirigidas Hacia las Mujeres. Resultados 1999-2009. Caracas, 2010.

Programa de Lucha de las Mujeres Patriotas y Revolucionarias. Documento Base para el Debate. Congreso Venezolano de Mujeres. 06,07 y 08 de Marzo de 2015. Caracas. 